Forum 2019 · 34:485

https://doi.org/10.1007/s12312-019-00684-z

Online publiziert: 16. September 2019

C) Springer Medizin Verlag $\mathrm{GmbH}$, ein Teil von

Springer Nature 2019

\section{Krebsgesellschaft}

Nordrhein-Westfalen e.V.

Krebsgesellschaft Nordrhein-Westfalen e.V.

Düsseldorf, Deutschland

\title{
Schülerinnen und Schüler brechen das Tabu!
}

\section{Krebsgesellschaft NRW ruft Schulen mit Videowettbewerb zu Kreativität gegen das Schweigen über Krebs auf}

deschluss für die Videos ist der 20.12.2019. Infos zur Teilnahme gibt es unter www. krebsgesellschaft-nrw.de.

\section{Film-Event zum Weltkrebstag 2020 im zakk Düsseldorf}

Eine Jury - bestehend aus Initiatoren und Unterstützern des Wettbewerbs - sichtet alle Beiträge und nominiert die zehn besten Klassen, Kurse, AGs für das finale Film-Event. Der Gewinner des Wettbewerbs wird dann am 3. Februar 2020 via Live-Juryentscheid im Düsseldorfer zakk gekürt. Auf großer Leinwand werden dort alle Werke gezeigt und die jeweiligen Film-Teams auf der Bühne interviewt.

Der Schulwettbewerb ist Teil der Kampagne „Sprich mit mir! Über Krebs.“, die in NRW seit 2018 läuft. Mit der Kampagne rückt die Krebsgesellschaft NRW gesellschaftliche Tabus und Sprachlosigkeit bei Krebs in den Mittelpunkt öffentlicher Diskussion. Herzstück ist das gleichnami- ge Buch. Ein Poetry-Slam mit Jean-Philippe Kindler im zakk setzte vergangenen Februar ein erstes kreatives Zeichen, der Wettbewerb mit finalem Filmfestival soll dies weiterführen.

Partner des Schulwettbewerbs und der Gesamtkampagne sind die AOK Rheinland/Hamburg, die KrebsStiftung NRW sowie die Fachgruppe Selbsthilfe.

\section{Weitere Informationen unter}

- https://www.krebsgesellschaftnrw.de/ sprichmitmir

- https://www.facebook.com/Krebsgesellschaft.NordrheinWestfalen

\section{Korrespondenzadresse}

\section{Dinah Oelschläger}

Krebsgesellschaft NRW

Volmerswerther Straße 20, 40221 Düsseldorf,

Deutschland

oelschlaeger@krebsgesellschaft-nrw.de für jeden funktioniert, aber Offenheit mit dem Thema kann helfen aufeinander zuzugehen", so Graeven. Diese Offenheit ist zentrales Thema des Wettbewerbs, der gezielt junge, heranwachsende Menschen anspricht. Kreativität kann ein Weg sein, einen offenen Dialog über das immer noch hartnäckige Tabu „Krebs“ zu fördern. Der landesweite Schülerwettbewerb lädt NRWKlassen, Kurse und AGs ab Stufe 10 ein, ihre Kreativität spielen zu lassen und ein Video zu drehen. Ob gefilmtes Theaterstück, Gedichtvortrag, Sketch oder Song, Kurzfilm oder TV-Spot, ob Zeichentrickoder Animationsfilm: der Umsetzungsform sind keine Grenzen gesetzt. Einsen-

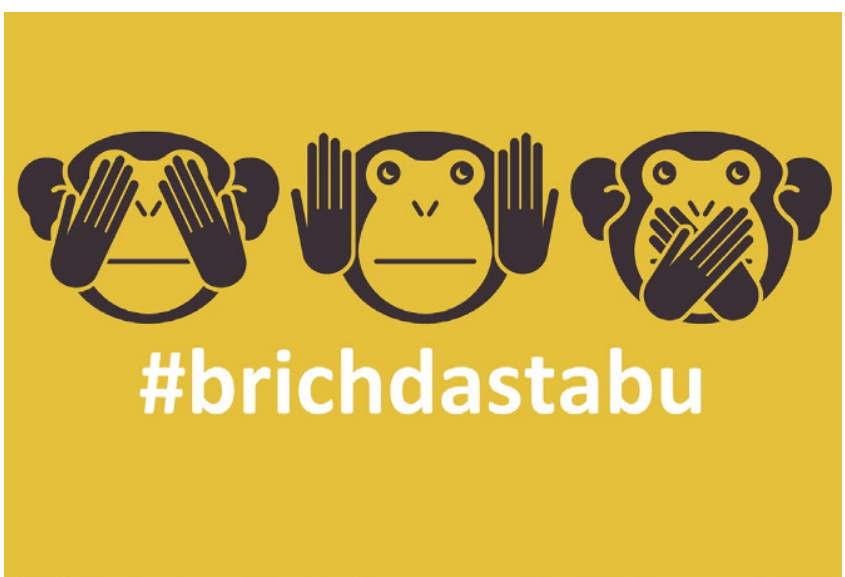

$\varangle(\odot$ RLRRLRLL) Adobe Stock) 